\title{
PREPARATION AND STUDY OF SINGLE SIZED CLUSTER IONS IN A SUPERSONIC JET
}

\author{
E. W. SCHLAG and H. L. SELZLE \\ Institut für Physikalische und Theoretische Chemie der Technischen \\ Universität München Lichtenbergstr. 4, D-8046 Garching
}

\begin{abstract}
Experiments with supersonic jet expansion have been performed to prepare clusters of benzene molecules. The clusters produced in the jet are ionized by two-photon ionization and mass analysis is achieved in a reflectron time of flight mass spectrometer. Due to an excess energy up to several hundred $\mathrm{meV}$ unimolecular decay occurs on a time-scale from nanoseconds up to microseconds and even longer. To discriminate against interferences from fragmentation of higher mass clusters in the observed spectra different experiments have been performed which include a new method by which single size cluster ions are stopped in the supersonic jet. These ions then stay isolated in space and the metastable decay of these complexes can be analyzed without further perturbation from fragmentation of higher clusters.
\end{abstract}

KEY WORDS: Cluster, ions, preparation, jet.

\section{INTRODUCTION}

Structure and dynamics of van der Waals cluster are subject of growing interest during the last years. The most useful synthesis of clusters is by rapid adiabatic expansion in a supersonic jet, where all cluster sizes are generated simultaneously. Size-selected production of neutral clusters is not yet possible beside some special cases, ${ }^{1}$ therefore the ionization process leads to a mixture of ions of different masses. The individual spectra of clusters of different sizes are mostly determined by ionization from a resonant intermediate state and separating of the spectra of different clusters by monitoring the ions of an individual size. This method suffers from the fact that due to the long onset of the ionization threshold ${ }^{2}$ one has to apply a second laser for ionization which is considerable above the ionization limit of the clusters and therefore fragmentation of the clusters becomes a major problem. This means that dissociation of from larger clusters contaminate the spectra of the lower sized clusters which can completely destroy the information of the parent molecule under observation.

Several methods of avoiding this problem can be considered. The first uses the effect of the excess energy onto the dissociation process, which implies that if one lowers the energy of the ionizing laser the amount of fragmentation is also lowered efficiently and should vanish if one reaches the ionization limit. ${ }^{3}$ Special care has to be taken to avoid multiphoton ionization with the second laser and therefore the intensity has to be kept low which in fact also diminishes the usable signal. 
The overlap of the long onset of the ionization potential especially for larger clusters does not allow for separation and therefore there will always be some excess energy and complete suppression of fragmentation cannot be accomplished.

Another method to discriminate against the contribution from fragmentation of higher clusters can be performed if one uses the fact that the fragmentation with excess energy leads to kinetic energy of the fragments. For this purpose the ionization is performed under field free conditions and extract them after some microseconds. The ions with kinetic energy from the dissociation process will move out of the region from which the ions are accepted from the time of flight (TOF) masspectrometer. This can be accomplished by reducing the steradiancy of the accepting angle with the use of small apertures in the path of the ions to the detector. It was found ${ }^{3}$ that the discrimination against nonresonant ionized clusters works efficiently as well as for fragments of ions prepared at larger excess energy which have a large amount of energy flowing in the intermolecular bond. For larger clusters this does not work very well, as the cluster ions mostly loose one neutral molecule in a single step. ${ }^{4}$ This takes with it most of energy as kinetic energy due to kinematic reasons. The change of the the momentum of the remaining larger fragment ion then is very small. This method therefore is only practicable for smaller clusters to clean the spectra from the background due to nonresonant multiphoton ionization.

To study the dissociation process of a cluster of a single size we now have introduced a new method for preparation and storing of monodisperse cluster ions in space. Here the fact is used that all clusters of different sizes are accelerated to the same speed during the supersonic expansion. This speed with a narrow distribution is maintained after laser ionization. Having the same speed the cluster ions are now well separated in momentum space and when one applies a retarding electric pulse which compensates the momentum of a cluster a single sized cluster can be brought to a complete stop, whereas all the other clusters leave the region of ionization. After a variable time delay during which the ions are available for further experiments the ions can be extracted into the reflectron time of flight (RETOF) mass spectrometer. The remaining mass spectrum thus contains only the stopped species and products which are generated after stopping the parent ion. This then allows for a unique determination of the kinetics of the fragmentation on a time scale from nanoseconds to microseconds. Varying the time before stopping of the ions after ionization a short time decay can be measured whereas the delay before extracting into the RETOF gives information on a longer time scale for the metastable decay of single size cluster ions.

\section{EXPERIMENTAL}

The supersonic jet experiment was described previously. ${ }^{5}$ Benzene at $10^{\circ} \mathrm{C}$ corresponding to 65 mbar seeded in $\mathrm{He}$ at 4 bar is expanded through a pulsed nozzle (modified Bosch fuel injection valve) into the vacuum. A distribution of neutral clusters with sizes $n>20$ at a reasonable amount is generated. A skimmer with a $1 \mathrm{~mm}$ opening placed $35 \mathrm{~mm}$ downstream of the nozzle selects the central part of the 
molecular beam. The beam of a frequency doubled dye lasers (QUANTA RAY PDL-1), pumped by a Nd:YAG (QUANTA RAY DCR1-A), is focused on the center of the jet. The ion optics consist of a repeller and an extractor plate, with center openings of $5.0 \mathrm{~mm}$ respectively, at a distance of $10 \mathrm{~mm}$. Copper meshes $(90 \%$ transmission) are mounted onto the openings to avoid lens effects. The ionization occurs under field free conditions with both plates on ground potential. When applying a proper pulse across the plates the ions can either be stopped or extracted into the RETOF. To stop a selected cluster a negative pulse of 300 volts and a duration of $80 \mathrm{nsec}$ to $500 \mathrm{nsec}$ is applied at the reflector and to extract the ions a positive voltage step of 900 volts at the repeller plate is used at a variable delay.

\section{RESULTS AND DISCUSSION}

In the supersonic jet expansion with benzene seeded into Helium as carrier gas clusters of benzene are formed at different sizes. The seed ratio was kept low and for this the speed of all clusters found is nearly the same as could be shown using a high speed chopper. ${ }^{6}$ The peaks of the momentum distribution

$$
p_{n}=m_{n} \cdot v
$$

with a width of

$$
\Delta p_{n}=m_{n} \cdot \Delta v
$$

are well separated, where $p_{n}$ is the momentum of the benzene cluster of the mass $m_{n}$ and $v$ is the average speed of all clusters, which amounts to $1500 \mathrm{~m} / \mathrm{sec}$ at a driving pressure of 2 bar of $\mathrm{He}$. The half width of the momentum distribution $\Delta p_{n}$ increases with the mass of the cluster as the width $\Delta v$ of the velocity distribution is nearly equal for all clusters studied and was found to be about $100 \mathrm{~m} / \mathrm{sec}$ at a driving pressure or 2 bar. Under our experimental conditions the ratio of the half width of the momentum distribution of the dimer to the spacing to the monomer and trimer is roughly 1:20 whereas this ratio is nearly 1:1 for clusters with $n$ in the order of 15 . The half width of the velocity distribution is a strong function of the driving pressure ${ }^{7}$ and makes it necessary to use very high pressure to separate large clusters in momentum space. It is also necessary to maintain a low seed ratio which unfortunately reduces the appearance of large clusters.

Figure 1 shows the time of flight mass spectrum for the case of small benzene clusters $(n<8)$. In this case the momentum distribution allows for complete separation of the cluster ion and stopping of a single sized cluster is achieved. In this case the benzene ${ }_{6}$ cluster was stopped and this cluster is the main feature in the mass spectrum obtained after $20 \mu \mathrm{sec}$ delay before extraction. The peak of the mass of benzene $_{5}$ arises from metastable decay and this technique allows for determination of the fragmentation kinetics by varying the time between stopping and extraction.

In Figure 2 the ion stopping is demonstrated in the region of larger clusters of benzene $(n>10)$. Here it is seen that the momentum distribution starts to overlap and is appreciable large already for benzene ${ }_{13}$. The mass spectrum without stopping 


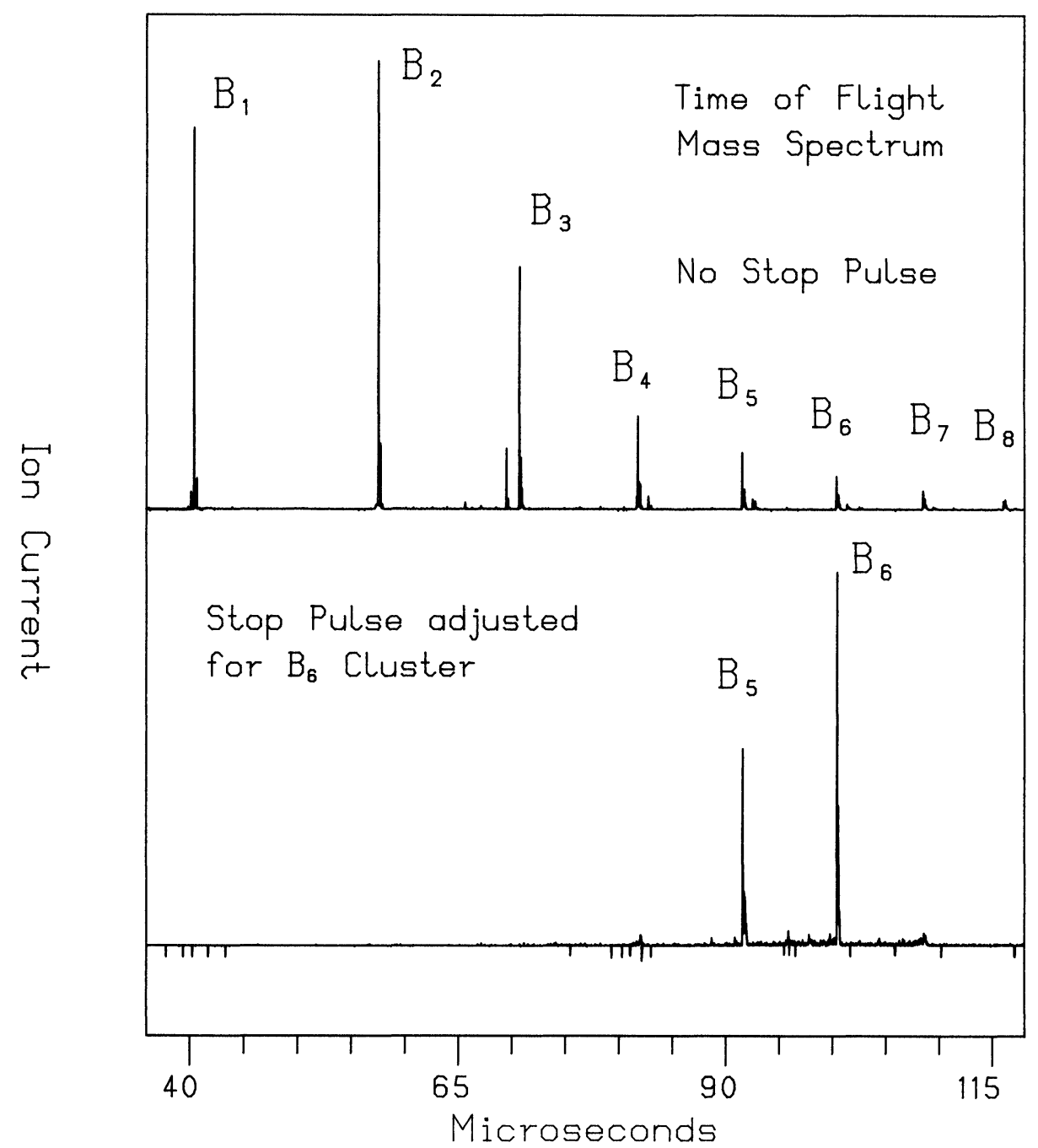

Figure 1 Stopping of a selected cluster of benzene ${ }_{6}$. The upper curve shows the mass spectrum if no stop pulse is applied and all ions are extracted immediately after ionization. The ionization laser is near the dimer $6_{0}^{1}$ transition to $S_{1}$. The lower mass spectrum was obtained by stopping the selected benzene ${ }_{6}$ cluster. The main peak arises from the stopped the parent ion and the lower peak originates from metastable decay.

shows a cluster distribution up to $n=21$. When a stopping pulse is applied which corresponds to the center of the momentum distribution of benzene ${ }_{13}$ the mass spectrum after a delay of $20 \mu \mathrm{sec}$ before extraction now also shows peaks for benzene $_{10}$ to benzene 14 . The intensity of these peaks is determined by the fragmentation of the clusters during the delay. In this case the metastable decay cannot directly be analyzed but the stopping here still has the advantage of selecting a narrow distribution of cluster ions $(\Delta n= \pm 1)$ as is seen from the only small contribution at the mass of benzene ${ }_{15}$. Thus the ion signal for benzene ${ }_{10}$ and 


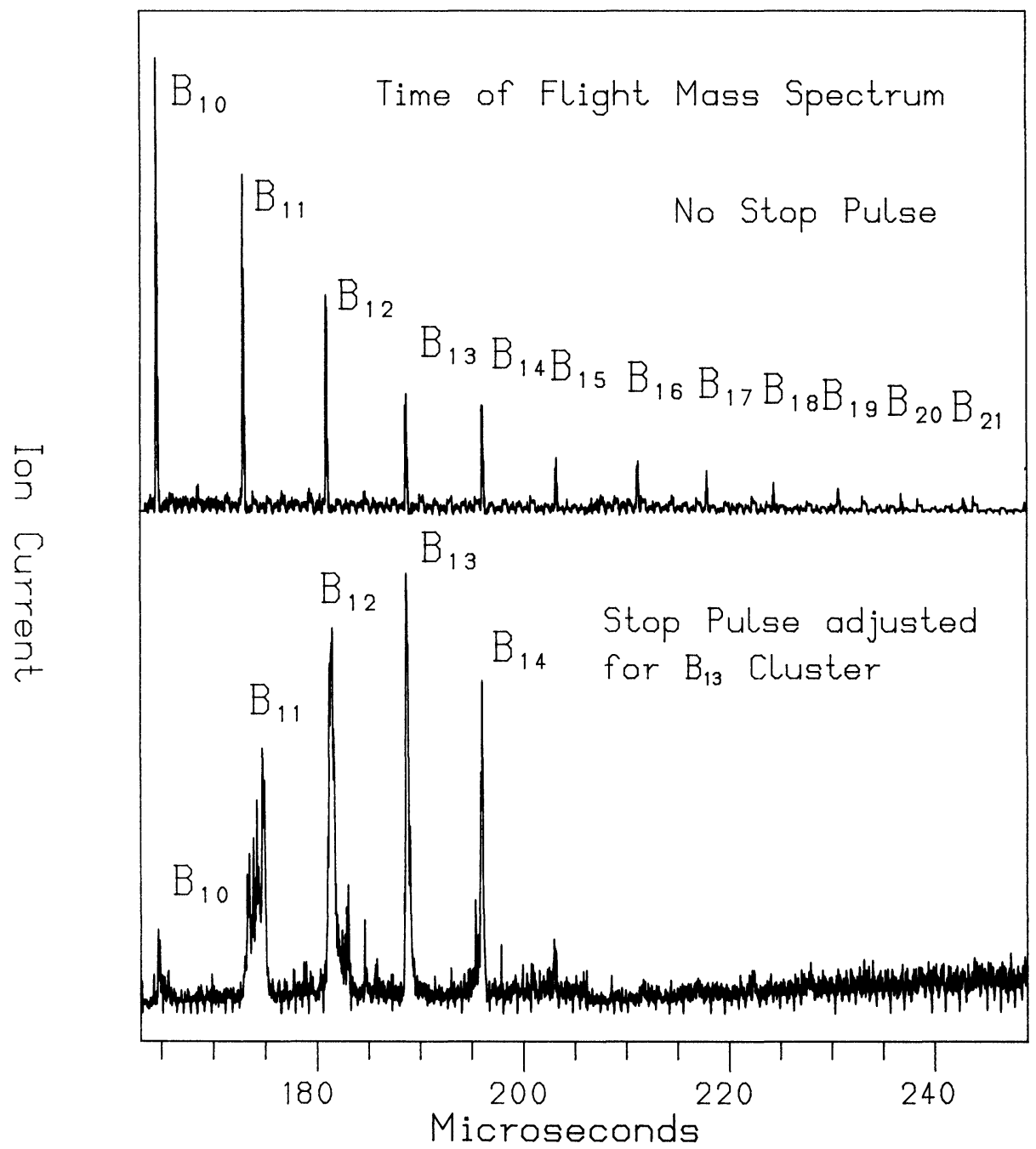

Figure 2 Stopping of selected clusters of benzene around the benzene ${ }_{13}$ cluster. The upper curve shows the mass spectrum if no stop pulse is applied and all ions are extracted immediately after ionization. The ionization laser was positioned near the $6_{0}^{1}$ transition of the benzene ${ }_{13}$ cluster. The lower mass spectrum was obtained by applying a stop pulse corresponding to the center of the momentum distribution of the benzene $_{13}$ cluster. In this case due to the larger width of the momentum distribution benzene $e_{12}$ and benzene $_{14}$ clusters are also stopped thus hiding the fragmentation contribution.

benzene $_{11}$ mostly arises from metastable decay; from varying the delay kinetic information of the metastable decay can also be obtained.

\section{CONCLUSION}

The preparation of molecular clusters of a single size is important for studying the stability and metastable decay of such complexes in order to get information about 
the amount of contamination of excitation spectra of neutral clusters with ionization detection. The method of stopping the clusters prepared in a supersonic jet allows for separation and storing of a single sized species. From varying the time before stopping and the delay before detection the kinetics of the metastable decay can be followed from nanosecond to the microsecond regime.

\section{References}

1. M. Arnold, J. Kowalski, G. zu Putlitz, T. Stehlin and F. Träger, Z. Phys. Teil A 322, 179 (1985).

2. S. H. Lin, H. L. Selzle, K. O. Börnsen and E. W. Schlag, J. Chem. Phys. 78, 309 (1983).

3. E. W. Schlag and H. L. Selzle, J. Chem. Soc. Faraday Trans. 86, 2511 (1990).

4. A. Kiermeier, B. Ernstberger, H. J. Neusser and E. W. Schlag, J. Phys. Chem. 92, 3785 (1988).

5. H. Bader, O. Krätzschmar, H. L. Selzle and E. W. Schlag, Z. Naturf. 44a, 1222 (1989).

6. S. Sengteller, H. L. Selzle and E. W. Schlag, Z. Naturf. 45a, 169 (1990).

7. J. Wang, V. A. Shamamian, B. R. Thomas, J. M. Wilkinson, J. Riley, C. F. Giese and W. R. Gentry, Phys. Rev. Lett. 60, 696 (1988). 